\title{
Comparative Actin Polymerization in Neonatal and Adult Bovine Neutrophils In Vitro
}

\author{
PHILIP N. BOCHSLER, NANCY R. NEILSEN, AND DAVID O. SLAUSON \\ Department of Pathobiology, College of Veterinary Medicine, The University of Tennessee, \\ Knoxville, Tennessee 37901
}

\begin{abstract}
Neutrophils rely on active reorganization of the cytoskeleton during movement, and functional deficiencies in the cytoskeletal elements may result in impaired neutrophil-mediated host defense. We have compared and quantitated actin polymerization in neonatal ( $\leq 48 \mathrm{~h}$ old $)$ and adult bovine peripheral-blood polymorphonuclear leukocytes (PMN) using fluorescence flow cytometry. Baseline filamentous actin (F-actin) content of neonatal and adult PMN at time zero differed slightly but were not statistically different $(p>0.05)$. F-actin content of recombinant human $\mathrm{C5a}\left(10^{-7} \mathrm{M}\right)$-stimulated neonatal PMN increased rapidly within $10 \mathrm{~s}$ of stimulation to $59.0 \%$ over baseline, then declined. F-actin in adult recombinant human C5a-stimulated PMN continued to increase for $30 \mathrm{~s}$ and was elevated $87.3 \%$ over baseline before subsequently declining. When stimulated with zymosan-activated bovine serum $(\mathbf{1 0} \%)$, neonatal (120.7\% increase) and adult PMN (115.1\% increase) had similar profiles with no significant differences, and both groups reached peak F-actin levels at $30 \mathrm{~s}$ after stimulation. Neonatal PMN stimulated with platelet-activating factor $\left(10^{-6} \mathrm{M}\right)$ attained peak F-actin values at $10 \mathrm{~s}(72.0 \%$ increase over baseline), but actin rapidly depolymerized by $30 \mathrm{~s}$ poststimulation (reduced to $29.0 \%$ increase). Adult PMN stimulated by platelet-activating factor also attained peak values by $10 \mathrm{~s}(97.6 \%$ increase over baseline), but in contrast to neonatal PMN the F-actin remained elevated at $30 \mathrm{~s}$ in the adult PMN (still increased $79.5 \% ; p<0.0 .5$ compared to neonatal Factin). Our results suggest that stimulus-specific variation may exist between responses of neonatal and adult bovine PMN to different agonists, but actin polymerization is rapid and PMN were stimulus-responsive in both age groups. (Pediatr Res 32: 509-513, 1992)
\end{abstract}

\section{Abbreviations}

F-actin, filamentous actin; polymerized actin PMN, polymorphonuclear leukocyte; neutrophil rhC5a, recombinant human $\mathrm{C5a}$

PAF, platelet-activating factor

ZAS, zymosan-activated bovine serum

RFU, relative fluorescence unit

HBSS-CM, Hanks' buffered salt solution containing $\mathrm{Ca}^{2+}$ and $\mathrm{Mg}^{2+}$

Circulating peripheral-blood leukocytes that respond to bloodborne pathogens or rapidly migrate to tissue sites of infection

Received April 17, 1992; accepted June 11, 1992

Correspondence and reprint requests: Dr. Philip N. Bochsler, Department of Pathobiology, College of Veterinary Medicine, The University of Tennessee, P.O. Box 1071, Knoxville, TN 37901-1071.

Supported by USDA competitive research Grant 90-37265-5611 and the University of Tennessee Centers of Excellence. provide one of the first lines of defense against invading organisms (1). PMN respond quickly and in large numbers to many stimuli and use potent microbicidal armament such as reactive oxygen species, phagocytosis, and degradative granule enzymes to disable microbial invaders (2). To respond to the presence of tissue invaders, the PMN execute a programmed series of events that include sensing a chemotactic agent or inflammatory stimulus, adhering to the vascular endothelium, migrating through the vessel wall, moving within the extracellular matrix of tissue toward the inflammatory focus, and finally reacting directly to the offending agent (3). The encounter of PMN with the agent may include attempted phagocytosis of particulate material, such as in the case of bacteria or fungi. Most of these PMN responses involve cellular movement or change of cellular shape, and dynamic involvement of the cellular cytoskeleton is requisite for movement of PMN (4). The predominant components of the cytoskeleton of PMN are actin microfilaments (5), which cycle between a nonpolymerized, monomeric form and a polymerized or filamentous form designated F-actin. These dynamic microfilaments contribute to plastic form and function of PMN.

Host defense of neonates is an area of considerable concern, and investigations have focused on the ability of neonatal PMN to effectively respond to inflammatory stimuli. PMN derived from human neonates have been reported to have diminished ability to up-regulate the integrin adhesion molecule Mac-1 (CD11b/CD18) $(6,7)$, comparable or decreased ability to generate antimicrobial reactive oxygen species (8), defective chemotaxis toward inflammatory signals (9), and variation in actin content as compared to PMN derived from adults $(9,10)$. Bovine neonatal PMN have been demonstrated to have a decreased respiratory burst $(11,12)$ and decreased $\mathrm{Fc}$ receptor expression. (13) when compared with adult bovine PMN. Alterations in the ability of neonatal PMN to mobilize actin, as reflected by actin polymerization and total cellular content of F-actin, may also be a contributing factor in diminished capability of PMN to perform movement-reliant operations such as chemotaxis, emigration out of blood vessels, and phagocytosis.

Because PMN are critical to effective host defense and need exists for a better understanding of factors contributing to the resistance of neonatal humans and animals to infectious agents, we have compared actin polymerization of neonatal and adult bovine PMN. An increase in cellular F-actin content, which is often concomitant with development of membrane ruffles and lamellipodia, is also of importance to PMN research because it is an early, sensitive indicator of PMN activation. Some differences were found in these studies in the magnitude of the response (i.e. total F-actin content) and kinetics of actin polymerization and depolymerization between neonatal and adult PMN, and our results also suggest that actin polymerization is rapid and responsive in PMN of both age groups.

\section{MATERIALS AND METHODS}

Neutrophil isolation. Blood $(30-60 \mathrm{~mL})$ was collected from clinically normal, colostrum-fed, newborn bovine calves within 
$48 \mathrm{~h}$ of birth and from nonparturient cows greater than $2 \mathrm{y}$ of age by jugular venipuncture using acid citrate dextrose $(1: 10)$ as the anticoagulant. Neutrophils were isolated by differential centrifugation combined with flash hypotonic lysis of the red blood cells as previously described $(11,14)$. Blood was centrifuged $(730$ $\times g$ for $15 \mathrm{~min}$ ), and the plasma, the buffy coat, and the top one half of the erythrocyte layer were removed and discarded. Erythrocytes were destroyed (hypotonic lysis) by addition of $20 \mathrm{~mL}$ of cold, sterile, pyrogen-free distilled water. Isotonicity was immediately restored by adding $10 \mathrm{~mL}$ of cold, sterile, pyrogen-free saline $(2.7 \% \mathrm{NaCl})$. Neutrophils underwent centrifugation $(200$ $\times g$ for $10 \mathrm{~min}$ ) and were washed twice with cold Hanks' buffered salt solution without $\mathrm{Ca}^{2+}$ and $\mathrm{Mg}^{2+}$. Final cell suspensions were diluted in HBSS-CM. Preparations of PMN used in these studies were composed of an average of $85.86 \pm 2.42 \%$ PMN for adults and $95.17 \pm 1.77 \%$ for neonates. The other leukocytes were primarily lymphocytes and eosinophils as determined by WrightGiemsa-stained cytocentrifuge preparations. Cell viability was high $(\geq 98 \%)$ as judged by trypan blue dye exclusion. Only samples with total and differential leukocyte counts and hematocrit values within established normal limits were used in these studies. Protocol for blood collection was approved by an animal care committee at this institution, and animals serving as blood donors received the highest level of care.

Labeling of polymerized actin and fluorescence flow cytometric analysis. PMN preparations in suspension $\left(3.0 \times 10^{6} / \mathrm{mL}\right.$ in HBSS-CM) were prewarmed for $5 \mathrm{~min}$ at $37^{\circ} \mathrm{C}$, then stimulated with rhC5a $\left(10^{-7} \mathrm{M}\right.$; Sigma Chemical Co., St. Louis, MO), PAF $\left(10^{-6} \mathrm{M}\right.$; Sigma), or ZAS (10\%; zymosan A, Sigma Chemical Co.), or unstimulated control cells were maintained in HBSSCM. rhC5a is a chemotactic agent with stimulatory effects on bovine PMN (15), as with human PMN. Some PMN were treated with $5 \mu \mathrm{g} / \mathrm{mL}$ of the microfilament-interfering agent cytochalasin $\mathrm{B}$ (Sigma Chemical Co.) for $10 \mathrm{~min}$ at $4^{\circ} \mathrm{C}$ before stimulation as a control. At time intervals of 0 (unstimulated PMN), 5, 10, 30, 120 , and $300 \mathrm{~s}$ after stimulation, the PMN were fixed with $1 \%$ paraformaldehyde at $22^{\circ} \mathrm{C}$ and gently mixed for $10 \mathrm{~min}$. PMN were centrifuged and washed with $\mathrm{HBSS}-\mathrm{CM}$, then resuspended in lysophosphatidylcholine $(100 \mu \mathrm{g} / \mathrm{mL}$; Sigma Chemical Co.) with Bodipy-labeled phallacidin $\left(10^{-8} \mathrm{M}\right.$; Molecular Probes, Eugene, OR) for $30 \mathrm{~min}$ at $4^{\circ} \mathrm{C}$ with constant slow mixing in the dark. The PMN were subsequently centrifuged, washed, and resuspended in PBS ( $\mathrm{pH}$ 7.3) for fluorescence flow cytometric analysis. Fluorescence analysis to detect $\mathrm{F}$-actin was performed using a Becton-Dickinson FACScan. Analysis gates quantitated the fluorescence associated with PMN, and 10000 cells were counted per treatment. Cellular autofluorescence was subtracted from relative fluorescence values before data analysis. Data were compared using absolute values of relative fluorescence (RFU) and the two-sample $t$ test (two-tailed) for comparisons between neonates and adults and the paired $t$ test for comparisons within age groups. Positive fluorescence stain of PMN was visually verified using Zeiss epifluorescence microscopy.

$Z A S$. Bovine blood was collected from multiple animals, allowed to clot, and centrifuged $(200 \times g$ for $15 \mathrm{~min})$. Serum was collected and pooled, and the alternate complement pathway activated by using zymosan $A$ at $2.0 \mathrm{mg} / \mathrm{mL}$ of serum, incubated for $30 \mathrm{~min}$ at $37^{\circ} \mathrm{C}$ with constant agitation (14). Serum was cooled to $4^{\circ} \mathrm{C}$, centrifuged for $10 \mathrm{~min}$ at $2000 \times g$, and decanted from the zymosan pellet. Serum was clarified by ultracentrifugation at $20000 \times g$ for $10 \mathrm{~min}$. The ZAS was tested for PMN stimulatory activity by aggregometry (16) and frozen at $-70^{\circ} \mathrm{C}$ until use.

\section{RESULTS}

Neonatal and adult bovine PMN stained avidly for F-actin with Bodipy phallicidin, but heterogeneity of staining intensity was observed within PMN populations in the same treatment groups. This may indicate differential ability to respond to stim- uli (i.e. mobilize the cytoskeleton), which could be due to subpopulations of PMN or may correspond to fluctuations in individual cellular F-actin content occurring nonharmoniously with the group of PMN. Mean fluorescence averages obtained with flow cytometry do not reflect this heterogeneity. The increased cell-associated fluorescence associated with agonist-stimulated PMN detected in these experiments was inhibitable with cytochalasin $\mathrm{B}$, indicating that fluorescence was related to F-actin content.

The baseline value of F-actin in neonatal and adult control PMN was similar at time zero (224.3 and $211.8 \mathrm{RFU}$, respectively), differing by only $5.9 \%$ (Fig. 1). Baseline F-actin values of neonatal and adult PMN also differed little $(p>0.05)$ at subsequent incubation time points of $5,10,30,120$, and $300 \mathrm{~s}$, with percent differences of $4.2 \%,{ }^{1} 12.6 \%,{ }^{1} 8.3 \%,{ }^{2} 6.0 \%,{ }^{2}$ and $4.9 \%{ }^{2}$ for these time points, respectively. Both neonatal and adult PMN responded rapidly to rhC5a, and cellular F-actin content rose quickly within $5 \mathrm{~s}$ of stimulation (Fig. 1). Neonatal PMN reached maximal actin polymerization at $10 \mathrm{~s}$ poststimulation $(59.0 \%$ increase over basal level, 459.8 RFU), and F-actin content declined progressively thereafter until content approached that of control cells by $300 \mathrm{~s}$ poststimulation. F-actin content of adult PMN was similar to that of neonates at $10 \mathrm{~s}$ poststimulation (481.21 RFU) but increased to maximal F-actin content at $30 \mathrm{~s}$ poststimulation ( $87.3 \%$ increase, $499.06 \mathrm{RFU})$. In contrast, Factin content was decreasing in neonatal PMN at $30 \mathrm{~s}$. The average maximal F-actin content of adult PMN was greater than that of neonatal PMN when expressed as a percentage of increase over baseline values and when expressed as absolute RFU, but there was no significant difference $(p>0.05)$ between data of neonatal and adult PMN at any time point when using rhC5a as a stimulus. Although increases for stimulated adult PMN were greater than those of neonates when expressed as a percentage of increase over baseline values, this was due in part to the slightly lower baseline F-actin content of adult PMN in the 0-, 5-, and 10 -s time points and corresponding higher baseline values of neonatal PMN (e.g. 211.77 RFU adult and 224.26 RFU neonate, at time zero). Baseline F-actin content of both neonatal and adult PMN (control/unstimulated PMN) also increased slightly during the incubation period at $37^{\circ} \mathrm{C}$, compared with $\mathrm{PMN}$ at time zero. Data were also acquired using ZAS (10\%), which contains complement fragments (e.g. C5a) as well as other serum factors, as a stimulus. The results using ZAS were qualitatively similar to those with purified rhC5a, except that neonatal PMN reached their peak $F$-actin content at $30 \mathrm{~s}$ poststimulation, as did adult PMN (Fig. 2).

The kinetics of actin polymerization when using PAF as a stimulus were different than those of the previous two stimuli (Fig. 3). Both neonatal and adult PMN reached maximal cellular F-actin content at only $10 \mathrm{~s}$ poststimulation, and net cellular actin depolymerization occurred beyond the 10-s time point. Peak F-actin values of neonatal PMN were $72.0 \%$ (497.37 RFU) greater than resting PMN, and values were $97.6 \%$ and 507.66 RFU for adult PMN. By 30 s poststimulation, F-actin in neonatal PMN had fallen to only $29.0 \%$ (317.30 RFU) above the baseline level. F-actin content in the adult PMN was still 79.5\% (478.24 RFU) above the baseline value at $30 \mathrm{~s}$ poststimulation, and the total cellular F-actin content of neonatal and adult PMN populations were significantly different $(p<0.05)$ at this time. However, by $120 \mathrm{~s}$ poststimulation, the F-actin content in both neonatal and adult PMN was low and nearly identical, and remained so at $300 \mathrm{~s}$ poststimulation.

Some variation was observed in the effect of the three different stimuli on actin polymerization in neonatal (Fig. $4 A$ ) and adult PMN (Fig. $4 B$ ). Preliminary investigations indicated that the concentrations of rhC5a $\left(10^{-7} \mathrm{M}\right)$, PAF $\left(10^{-6} \mathrm{M}\right)$, and ZAS $(10 \%)$ were maximal stimulatory concentrations for bovine

\footnotetext{
Neonate value higher.

Adult value higher.
} 


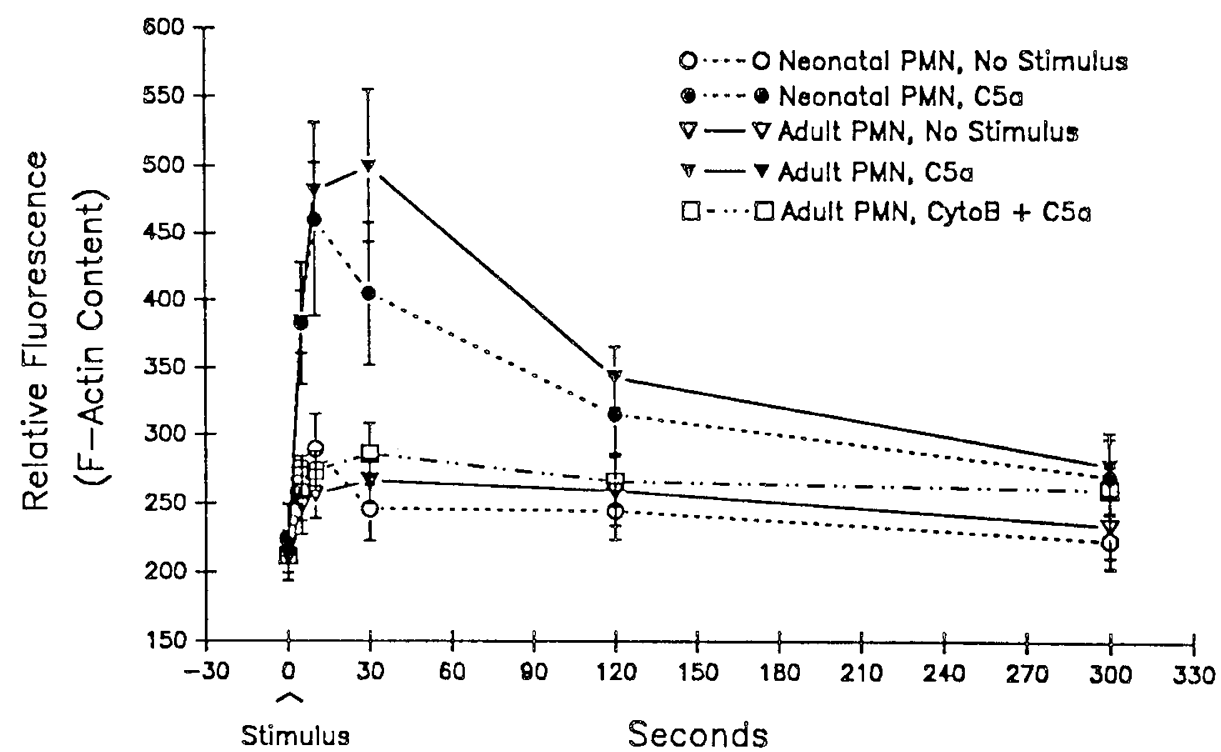

Fig. 1. Comparison of rhC5a-induced actin polymerization in neonatal and adult bovine PMN. Time-dependent F-actin content is shown at time zero and at 5,10,30,120, and $300 \mathrm{~s}$ after stimulation with $10^{-7} \mathrm{M}$ rhC5a. Values are means $\pm \mathrm{SEM} ; n=6$ neonates and $n=7$ adults.

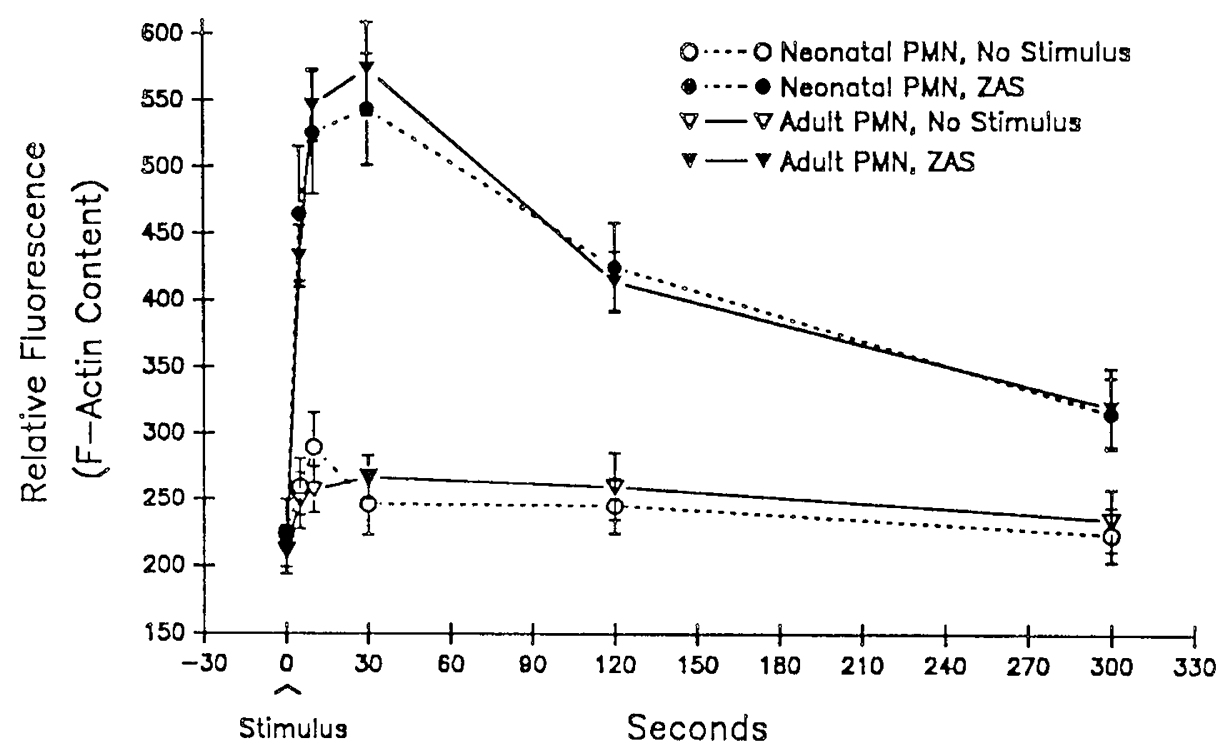

Fig. 2. ZAS-induced actin polymerization in neonatal and adult bovine PMN. Content of F-actin is shown at time zero and after stimulation with $10 \%$ ZAS. Values are means \pm SEM; $n=6$ neonates and $n=7$ adults.

$\mathrm{PMN}$, but definitive comparisons of stimuli require expanded dose-response studies to be conclusive.

\section{DISCUSSION}

An important cause of mortality of human and animal neonates is bacterial infection, and increased susceptibility of newborns to infection may be due to developmental defects or immaturity of components of the immune system (17-19). Human neonatal PMN have been reported to have deficiencies that could impair host defense $(6,8,9)$, including abnormalities in chemotaxis $(20,21)$ and differences in neonatal PMN actin polymerization (10) and microtubule organization (22). These latter observations may help to explain diminished ability of human neonatal PMN to undergo chemotaxis and orientation responses similarly to adult PMN. Like human newborns, bovine neonatal calves are also susceptible to a variety of bacterial infections (19). Comparative research of bovine PMN from neonates and adults also reports some functional differences. Neonatal bovine PMN generate decreased superoxide anion as compared to adult PMN with the protein kinase $\mathrm{C}$ activator phorbol myristate acetate $(11,12)$, but there is an increased neonatal PMN response to opsonized zymosan (14). Neonatal bovine PMN stimulated with zymosan-activated plasma undergo cellular shape change more rapidly and to a greater extent than adult PMN, as defined by development of membrane ruffles and lamellipodia within a percentage of the total PMN population (14). The ability of bovine PMN to aggregate in vitro is similar between the two age groups with either PAF or activated plasma as stimuli, but plasma-stimulated neonatal PMN had limited ability to deaggregate $(23,24)$. We undertook these studies to determine whether detectible differences exist between neonatal and adult bovine PMN in the kinetics and extent of actin polymerization.

Our data indicate that the kinetics of actin polymerization, as interpreted from total cellular F-actin content was, in general, similar between neonatal and adult bovine PMN. The data failed to demonstrate a remarkably deficient response from neonatal bovine PMN. The results corroborate with the reported chemotactic response of neonatal bovine PMN toward C5a, zymosanactivated plasma, and endotoxin as measured in microchemo- 


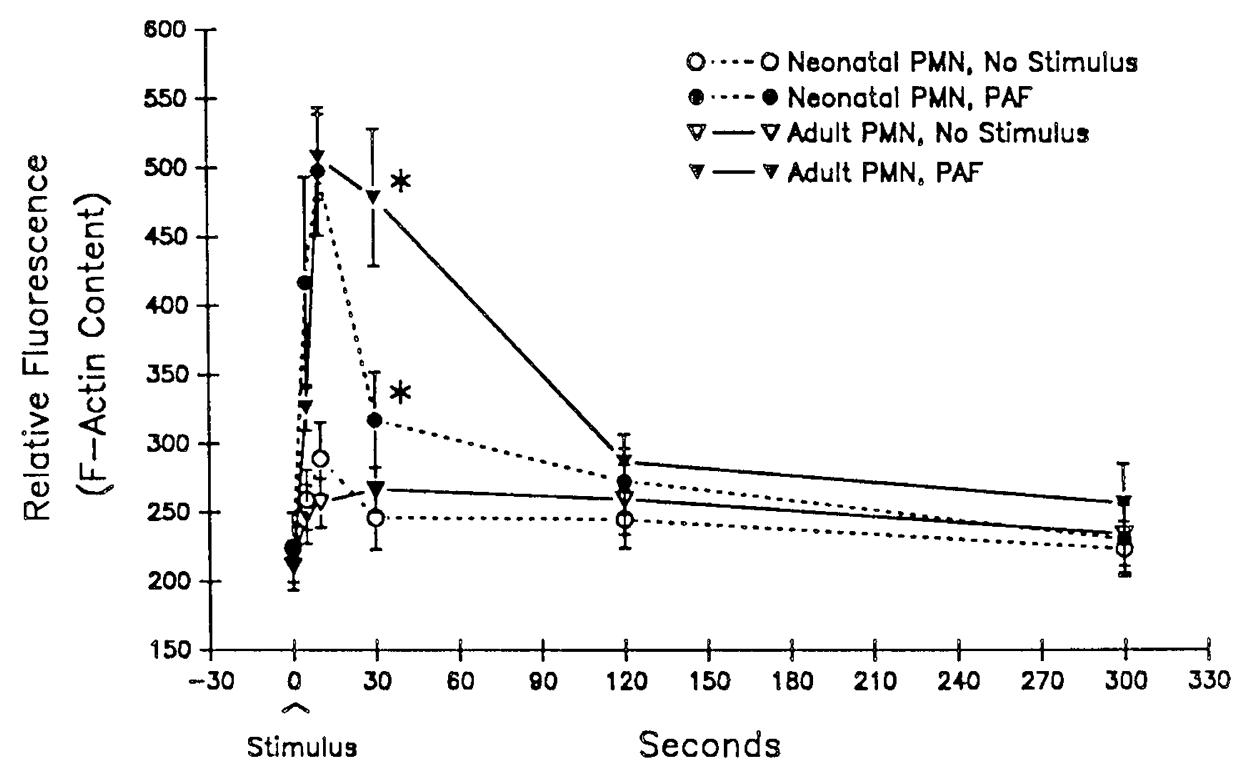

Fig. 3. Actin polymerization in neonatal and adult bovine PMN after stimulation with $\operatorname{PAF}\left(10^{-6} \mathrm{M}\right)$. An asterisk indicates significant difference $(p<0.05)$ between these two points. Values are means \pm SEM; $n=6$ neonates and $n=7$ adults.

A

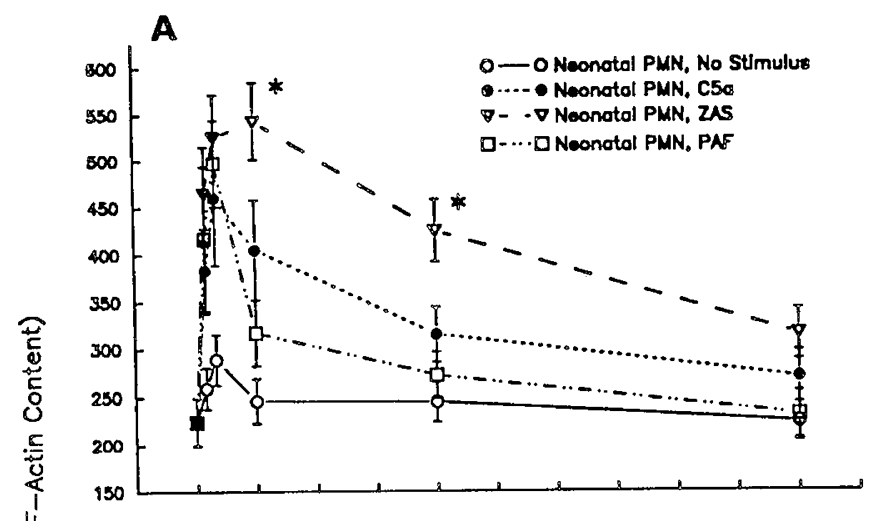

B

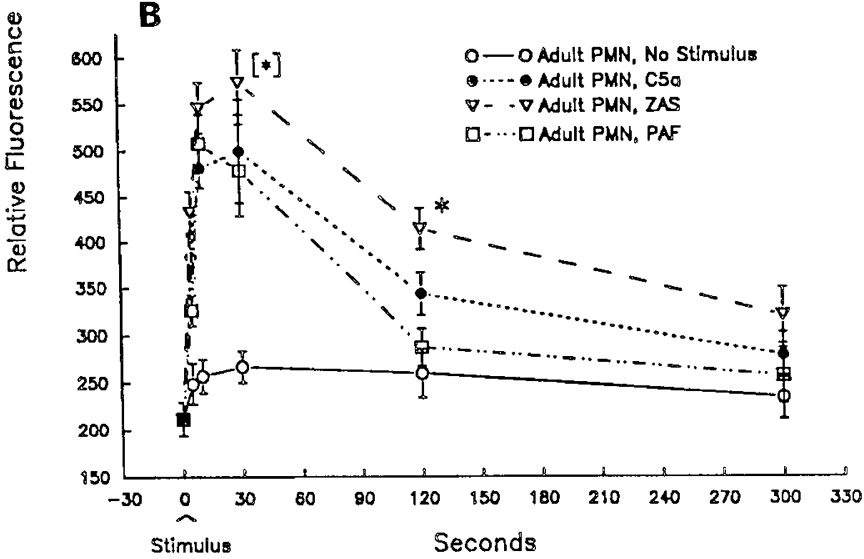

Fig. 4. $A, \mathrm{~F}$-actin data acquired using different stimuli with neonatal bovine PMN are compared on the same graph; rhC5a $\left(10^{-7} \mathrm{M}\right), \mathrm{ZAS}$ $(10 \%)$, and PAF $\left(10^{-6} \mathrm{M}\right)$. An asterisk indicates significant difference $(p$ $<0.05$ ) between these data points and all other data at the same time point. Values are means $\pm \mathrm{SEM} ; n=6$. A comparison appears in $B$ for adult PMN. An asterisk in brackets indicates that values for ZAS and PAF (and control) are significantly different $(p<0.05)$ at $30 \mathrm{~s}$ (values for ZAS and C5a are not). An asterisk with no brackets indicates significant difference $(p<0.05)$ between ZAS data and all other data at $120 \mathrm{~s}$. Values are means $\pm \mathrm{SEM} ; n=7$. taxis chambers by other investigators (23). Neonatal bovine PMN did not display impaired chemotaxis and instead migrated in greater numbers than did adult PMN. Similarly, in a study comparing shape change of stimulated neonatal and adult bovine PMN, investigators found that greater numbers of neonatal PMN underwent a more rapid change than adult PMN (14), which was maintained throughout the 5 -min incubation period. Shape change of PMN refers to rapid, stimulus-induced development of membrane ruffles and lamellipodia and occurs concomitantly or subsequent to actin polymerization in PMN (25). Our results in this study with F-actin do not demonstrate increased F-actin content of bovine neonatal PMN that might correlate with the other reports of neonatal bovine PMN response. In another study, investigators report that neonatal bovine PMN mobilize calcium as efficiently as adult PMN (15). This is in contrast to data from human PMN, where significant differences have been reported between the age groups for free intracellular calcium after stimulation, chemotaxis, and F-actin content (9). For each of these parameters, response of human neonatal PMN was less than adult PMN, contrary to findings with neonatal bovine PMN. Other investigators have found that F-actin content of stimulated human neonatal and adult PMN were similar, but that neonatal PMN have higher baseline F-actin content (10). Comparison indicates that species variation exists between responses of neonatal human and bovine PMN, and this may reflect innate differences or differences in the perinatal environment.

The cytoskeleton of PMN is a necessary component of directed cellular movement and is therefore important for normal function of PMN in host defense. Our results in this study of actin polymerization and other studies conducted by researchers who have investigated neonatal bovine PMN chemotaxis $(23,26)$ do not indicate that hypofunction of the neonatal bovine PMN actin network is likely to be a predisposing factor in susceptibility of newborn calves to microbial pathogens.

\section{REFERENCES}

1. Colditz IG, Kerlin RL, Watson DL 1988 Migration of neutrophils and their role in elaboration of host defense. In: Husband AJ (ed) Migration and Homing of Lymphoid Cells. CRC Press, Boca Raton, FL, pp 135-165

2. Sawyer DW, Donowitz GR, Mandell GL 1989 Polymorphonuclear neutrophils: an effective antimicrobial force. Rev Infect Dis 11(suppl 7): S1532-S1544

3. Van Kessel KPM, Verhoef J 1990 A view to a kill: cytotoxic mechanisms of 
human polymorphonuclear leukocytes compared with monocytes and natural killer cells. Pathobiology 58:249-264

4. Stossel TP 1989 From signal to pseudopod. How cells control cytoplasmic actin assembly. J Biol Chem 264:18261-18264

5. Korn ED 1982 Actin polymerization and its regulation by proteins from nonmuscle cells. Physiol Rev 62:672-737

6. Anderson DC, Becker Freeman KL, Heerdt B, Hughes BJ, Jack RM, Smith CW 1987 Abnormal stimulated adherence of neonatal granulocytes: impaired induction of surface Mac-1 by chemotactic factors or secretagogues. Blood 70:740-750

7. Smith JB, Kunjummen RD, Raghavender BH 1991 Eosinophils and neutrophils of human neonates have similar impairments of quantitative upregulation of Mac-1 (CD $1 \mathrm{lb} / \mathrm{CD} 18)$ expression in vitro. Pediatr Res 30 355-361

8. Kugo M, Sano K, Uetani Y, Nakamura H 1989 Superoxide dismutase in polymorphonuclear leukocytes of term newborn infants and very low birth weight infants. Pediatr Res 26:227-231

9. Sacchi F, Augustine NH, Coello MM, Morris EZ, Hill HR 1987 Abnormality in actin polymerization associated with defective chemotaxis in neutrophils from neonates. Int Arch Allergy Appl Immunol 84:32-39

10. Hilmo A, Howard TH $1987 \mathrm{~F}$-actin content of neonate and adult neutrophils. Blood 69:945-949

11. Clifford CB, Slauson DO, Neilsen NR, Suyemoto MM, Zwahlen RD, Schlafer DH 1989 Ontogeny of inflammatory cell responsiveness: superoxide anion generation by phorbol ester-stimulated fetal, neonatal, and adult bovine neutrophils. Inflammation 13:221-231

12. Doré M, Slauson DO, Neilsen NR 1992 Decreased respiratory burst activity in neonatal bovine neutrophils stimulated by protein kinase $\mathrm{C}$ agonists. Am J Vet Res 52:375-380

13. Zwahlen RD, Wyder-Walther M, Roth DR 1992 FC receptor expression, concanavalin A capping, and enzyme content of bovine neonatal neutrophils a comparative study with adult cattle. J Leukocyte Biol 51:264-269

14. Holden W, Slauson DO, Zwahlen RD, Suyemoto MM, Doré M, Neilsen NR 1989 Alterations in complement-induced shape change and stimulus-specific superoxide anion generation by neonatal calf-neutrophils. Inflammation 13 $607-620$

15. Doré M, Slauson DO, Suyemoto MM, Neilsen NR 1990 Calcium mobilization in C5a-stimulated adult and newborn bovine neutrophils. Inflammation 14 $71-82$

16. Hammerschmidt DE, Bowers TK, Lammi-Keefe CJ, Jacob HS, Craddock PR 1980 Granulocyte aggregometry: a sensitive technique for the detection of C5a and complement activation. Blood 55:898-902

17. Wilson CB 1986 Immunological basis for increased susceptibility of the neonate to infection. J Pediatr 108:1-12

18. Hill HR 1987 Biochemical, structural, and functional abnormalities of polymorphonuclear leukocytes in the neonate. Pediatr Res 22:375-382

19. Bryson DG, McFerran JB, Ball HJ, Neill SD 1978 Observations on outbreaks of respiratory disease in housed calves. 1. Epidemiological, clinical and microbiological findings. Vet Rec 103:485-489

20. Klein RB, Fisher TJ, Gard SE, Biberstein M, Rich KC, Stiehm RE 1977 Decreased mononuclear and polymorphonuclear leukocyte chemotaxis in human newborns, infants and young children. Pediatrics 60:467-472

21. Sacchi F, Rondini G, Mingrat G, Stronati M, Gancia GP, Marseglia GL, Siccardi AG 1982 Different maturation of neutrophil chemotaxis in term and pre-term newborn infants. J Pediatr 101:273-274

22. Anderson D, Hughes BJ, Wible L, Perry G, Smith C, Brinkly B 1984 Impaired motility of neonatal PMN leukocytes: relationship to abnormalities of cell orientation and assembly of microtubules in chemotactic gradients. J Leukocyte Biol 36:1-15

23. Zwahlen RD, Roth DR 1990 Chemotactic competence of neutrophils from neonatal calves. Functional comparison with neutrophils from adult cattle. Inflammation 14:109-123

24. Zwahlen RD, Roth DR, Wyder-Walther M 1990 In vitro aggregation of bovine neonatal neutrophils. A comparative study with adult cattle. Inflammation 14:375-387

25. Watts RG, Crispens MA, Howard TH 1991 A quantitative study of the role of F-actin in producing neutrophil shape. Cell Motil Cytoskeleton 19:159-168

26. Roth DR, Zwahlen RD 1991 Intradermal neutrophil mobilization induced by complement fragments and endotoxin is more effective in neonatal than adult cattle. Vet Pathol 28:446-448 